\title{
PROPAGATION OF ENERGETIC ELECTRONS FROM THE CORONA INTO INTERPLANETARY SPACE AND TYPE III RADIO EMISSION
}

\author{
F. Breitling*, G. Mann*, and C. Vocks*
}

\begin{abstract}
During solar flares a large amount of electrons with energies greater than $20 \mathrm{keV}$ is generated with a production rate of typically $10^{36} \mathrm{~s}^{-1}$. A part of them is able to propagate along open magnetic field lines through the corona into interplanetary space. During their travel they emit radio radiation which is observed as type III radio bursts in the frequency range from $100 \mathrm{MHz}$ down to $10 \mathrm{kHz}$ by the WAVES radio spectrometer aboard the spacecraft WIND, for instance. From the drift rates of these bursts in dynamic radio spectra the radial propagation velocity $V_{r}$ of the type III burst exciting electrons is derived by employing a newly developed density model of the heliosphere. Calculations show that the radio radiation is emitted by electrons with different $V_{r}$ and therefore by different electrons of the initially produced electron distribution.
\end{abstract}

\section{Introduction}

During solar flares a large amount of energetic non-thermal electrons, i.e. with energies greater than $20 \mathrm{keV}$ is generated with a production rate of typically $10^{36} \mathrm{~s}^{-1}$ [Warmuth et al., 2009]. About 0.1 to $1 \%$ of them [Lin and Hudson, 1971] are able to propagate along open magnetic field lines through the corona into interplanetary space. Signatures of these electrons can be observed as so called type III bursts in dynamic radio spectra [Suzuki and Dulk, 1985; Gurnett, 1995]. At a greater distance, the velocity distribution of the electrons develops a bump as shown in Fig. 1, since slower electrons have not yet arrived and the number of faster electrons decreases with energy. For comparison Fig. 1 also shows the distribution of thermal electrons in the corona, i.e. the energetic tail. Non-thermal electron bumps cause so called "bump on tail" instabilities which excite Langmuir waves, plasma emission and eventually radio radiation [Krall and Trivelpiece, 1973].

\footnotetext{
* Astrophysikalisches Institut Potsdam, An der Sternwarte 16, 14482 Potsdam, Germany
} 


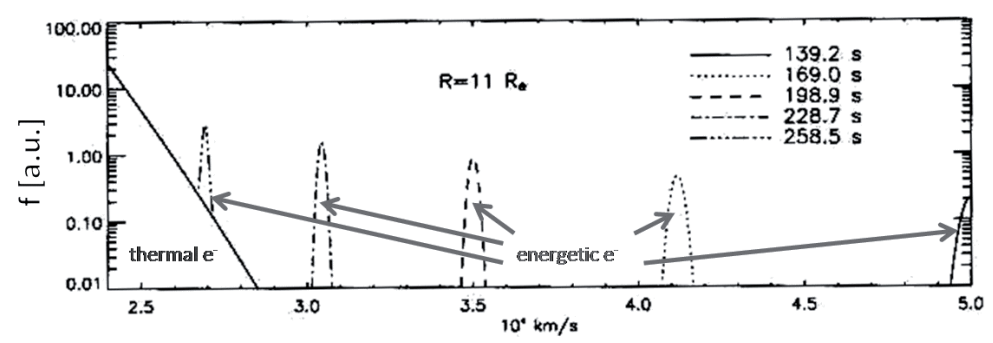

Figure 1: Velocity distribution $f(v)$ of thermal and non-thermal energetic electrons in a plasma which cause a "bump on tail" instability. Courtesy by Estel [1999].

Fig. 2 shows the typical spectrum of one type II and at least three type III bursts. In the upper two plots one sees the energetic radiation recorded by the X-ray satellites GOES and RHESSI [Lin et al., 2002]. In the lower two plots one sees the dynamic radio spectra recorded by the Observatory for Solar Radio Astronomy (OSRA) of the Astrophysical Institut Potsdam [Mann et al., 1992] and the WIND satellite [Bougeret, 1995]. The steep edge and the frequency drift of the radio emission from higher to lower frequencies is a prominent feature of type III bursts which is most apparent in the WIND spectrum. Since the radio radiation originates from plasma oscillations the emission frequency is directly

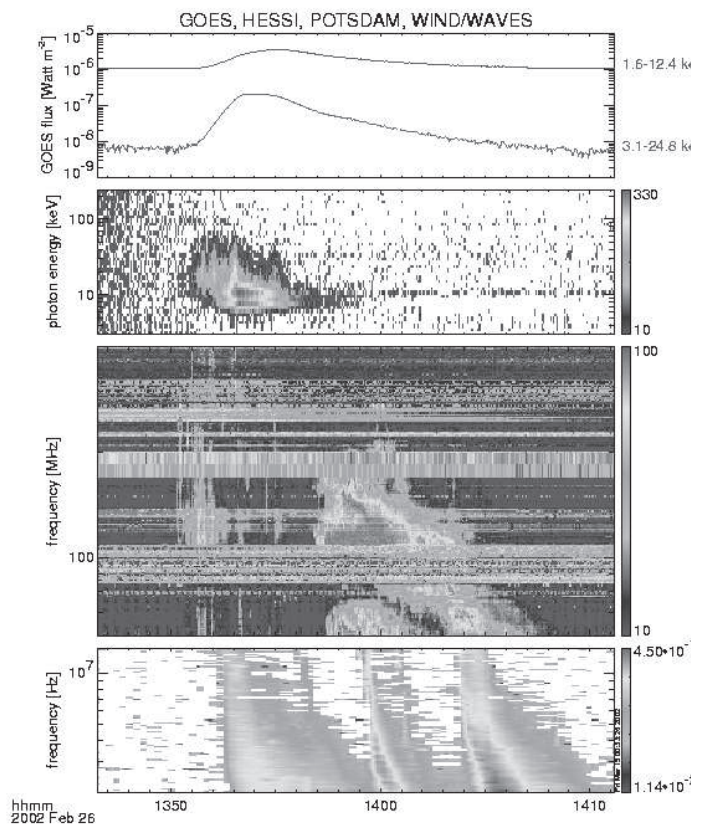

Figure 2: Dynamic spectra of one type II and at least three type III radio bursts recorded on 2002 Feb 26 by the X-ray satellites GOES and RHESSI, the Observatory for Solar Radio Astronomy (OSRA) and the WIND satellite in subsequent order from top to bottom. The type III burst are identified by their steep edge and the frequency drift of the radio emission from higher to lower frequencies best visible in the WIND spectrum. 
determined by the plasma density. The density and therefore the plasma frequency decreases with increasing distance to the Sun due to the Suns gravitation. Consequently the radio radiation from radially propagating electrons drifts from higher to lower frequencies. This has been recorded for decades in OSRA radio spectra. With the new radio interferometer LOFAR (LOw Frequency ARray) radio spectra of higher sensitivity and spectral resolution become available in the frequency range from 30 to $240 \mathrm{MHz}$. For example for spectroscopy the effective area increases from about $3 \mathrm{~m}^{2}$ to $96 \times 3 \mathrm{~m}^{2}$ and the spectral resolution improves from 300 to $3 \mathrm{kHz}$. Moreover LOFAR's imaging capabilities allow for the spatial resolution of burst propagation. So both instruments provide opportunities to study the spectra form type III burst in more detail and allow to model the electron propagation based on the theoretical understanding. Since electron propagation plays a key roll in space weather its understanding allows for more accurate space weather predictions which motivates further investigation.

\section{Drift Rates and Radial Propagation Velocity}

Plasma emission occurs at the fundamental plasma frequency $(n=1)$ and / or its first harmonic $(n=2)$ [Melrose, 1985]

$$
f=\frac{n}{2 \pi} \sqrt{\frac{e^{2} N_{\mathrm{e}}}{\epsilon_{0} m_{\mathrm{e}}}}
$$

where $\epsilon_{0}$ is the vacuum permittivity, $e$ and $m_{\mathrm{e}}$ are the charge and mass of the electron and $N_{\mathrm{e}}$ is the plasma's electron density. Since all other parameters are physical constants, $f$ is a function of $N_{\mathrm{e}}$ only.

The drift rate $D_{f}$ is the frequency change $f$ over time $t$. Using equation (1) one finds

$$
D_{f}=\frac{\mathrm{d} f}{\mathrm{~d} t}=\frac{\mathrm{d} f}{\mathrm{~d} N_{\mathrm{e}}} \frac{\mathrm{d} N_{\mathrm{e}}}{\mathrm{d} r} \frac{\mathrm{d} r}{\mathrm{~d} t}=\frac{f}{2 N_{\mathrm{e}}} \frac{\mathrm{d} N_{\mathrm{e}}}{\mathrm{d} r} \frac{\mathrm{d} r}{\mathrm{~d} t},
$$

where $r$ is the radial distance from the center of the Sun. Consequently the term $d r / d t$ expresses a radial propagation velocity and will be abbreviated by $V_{r}$. Then rewriting equation (2) yields an expression for the radial propagation velocity

$$
V_{r}=\frac{2 D_{f}}{f} N_{\mathrm{e}}\left(\frac{\mathrm{d} N_{\mathrm{e}}}{\mathrm{d} r}\right)^{-1}
$$

This equation shows, that measurements of the emission frequency and the drift rate as well as knowledge of the electron density of the interplanetary space allows to determine the radial propagation velocity $V_{r}$ of the radio radiation emitting electrons. 


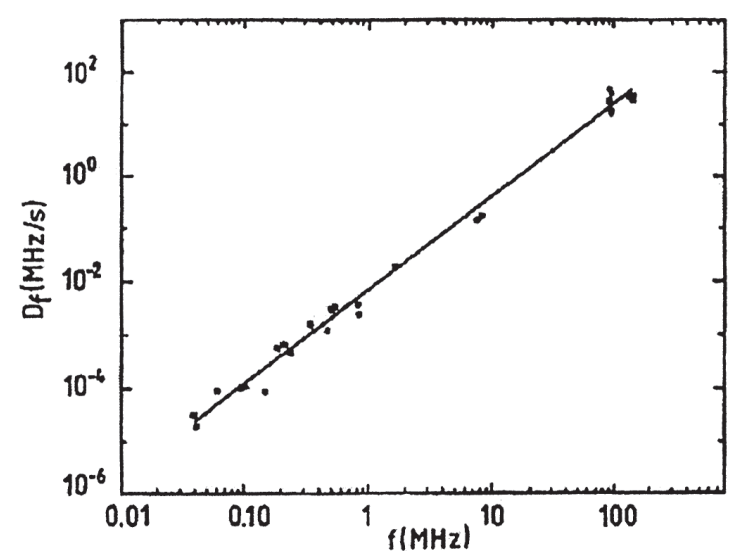

Figure 3: Drift rate of a type III radio burst on December 27, 1994 as determined by Mann et al. [1999] using measurements from OSRA, WIND and the ULYSSES satellite. The fit illustrates the power-law relation of equation (4).

Drift rates can be determined quite accurately from dynamic radio spectra. A typical example is show in Fig. 3 which was taken from Mann et al. [1999]. It shows that the drift rate, which has been determined at the leading edge of the type III burst signature, obeys the power-law

$$
D_{f}=-7.354 \times 10^{-3} \frac{\mathrm{MHz}}{\mathrm{s}}\left(\frac{f}{\mathrm{MHz}}\right)^{1.76} .
$$

However, on small scales and in particular at higher frequencies above $80 \mathrm{MHz}$ deviations from a power-law are possible.

\section{Density Model for the Interplanetary Space}

According to equation (3) the heliospheric electron density is required for determining the radial propagation velocity $V_{r}$ from drift rate measurements. The electron density is directly proportional to the mass density of the interplanetary plasma $N$ since $N_{\mathrm{e}}=$ $N /\left(1.92 \tilde{\mu} m_{\mathrm{p}}\right)$, where $m_{\mathrm{p}}$ is the proton mass and $\tilde{\mu}$ is the mean molecular weight [Priest, 1982]. In the inner corona up to a distance of 1.6 solar radii $N$ has been determined from optical measurements [Newkirk, 1961; Koutchmy, 1994; Warmuth et al., 2005] From 0.3 to $1 \mathrm{AU} N$ has been measured by the HELIOS satellite [Schwenn, 1990]. Unfortunately it hasn't been measured in the region from 1.6 solar radii to $0.3 \mathrm{AU}$. However, one can apply laws of physics to model the density there. For simplicity we neglect local density variations and consider mean radially symmetric models only. For example Mann et al. [1999] have applied the Parker [1958] wind model to data from the Helios satellite to 
obtain an interplanetary density model. Ansatz is the equation of motion

$$
m N v \frac{\mathrm{d} v}{\mathrm{~d} r}=-\frac{\mathrm{d} p}{\mathrm{~d} r}-\frac{G M_{\odot} m N}{r^{2}},
$$

where $m, N$ and $v$ are the mean particle mass, density, and velocity in radial direction $r$, $p$ is the pressure, $G$ is the gravitational constant and $M_{\odot}$ is the solar mass.

Here this model is extended by adding heat conduction as well as heating and pressure by Alfvén waves as done by Hackenberg et al. [2000].

Heat conduction is added via the energy equation

$$
v N\left[\frac{3}{2} k_{B} \frac{\mathrm{d} T}{\mathrm{~d} r}-k_{B} \frac{T}{n} \frac{\mathrm{d} n}{\mathrm{~d} r}\right]=\frac{1}{r^{2}} \frac{\mathrm{d}}{\mathrm{d} r}\left(r^{2} \kappa_{0} \frac{\mathrm{d} T}{\mathrm{~d} r}\right)+H,
$$

where $k_{B}$ is the Boltzmann constant, $T$ is the temperature, $\kappa_{0}$ is the thermal conductivity and $H$ is the heating function by the Alfvén waves.

$$
H(r)=-P_{0}\left[v(r)+v_{\mathrm{A}}(r)\right] \frac{1}{\omega_{\mathrm{H}}} \frac{\frac{v\left(R_{\odot}\right)}{v_{\mathrm{A}}\left(R_{\odot}\right)}\left(1+\frac{v\left(R_{\odot}\right)}{v_{\mathrm{A}}\left(R_{\odot}\right)}\right)^{2}}{\frac{v(r)}{v_{\mathrm{A}}(r)}\left(1+\frac{v(r)}{v_{\mathrm{A}}(r)}\right)^{2}} \frac{\partial \omega_{\mathrm{H}}}{\partial r},
$$

where $P_{0}$ is a pressure parameter, $\omega_{\mathrm{H}}$ is the higher cut-off frequency, $v_{\mathrm{A}}$ is the Alfvén velocity and $R_{\odot}$ is the solar radius.

The Alfvén wave pressure $p_{\mathrm{A}}$ adds the term $-\mathrm{d} p_{\mathrm{A}} / \mathrm{d} r$ to the momentum equation (5), where

$$
p_{\mathrm{A}}(r)=\frac{1}{2} P_{0} \log \left(\frac{\omega_{\mathrm{H}}}{\omega_{\mathrm{L}}}\right) \frac{\frac{v\left(R_{\odot}\right)}{v_{\mathrm{A}}\left(R_{\odot}\right)}\left(1+\frac{v\left(R_{\odot}\right)}{v_{\mathrm{A}}\left(R_{\odot}\right)}\right)^{2}}{\frac{v(r)}{v_{\mathrm{A}}(r)}\left(1+\frac{v(r)}{v_{\mathrm{A}}(r)}\right)^{2}},
$$

and $\omega_{\mathrm{L}}$ is the lower cut-off frequency.

The result is shown in Fig. 4 and table 1. Table 1 shows good agreement between the new density model and existing data. Fig. 5 shows good agreement of this model with density measurements of the inner corona.

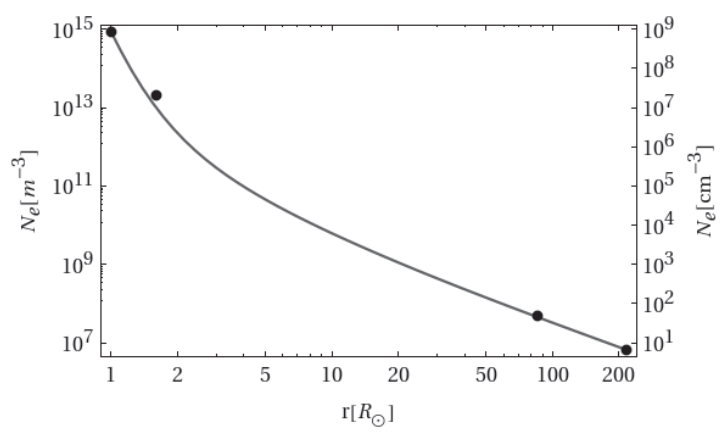

Figure 4: Electron density of the interplanetary space as known from measurements (four data points) and the new model described here. For numerical values and references see table 1. 


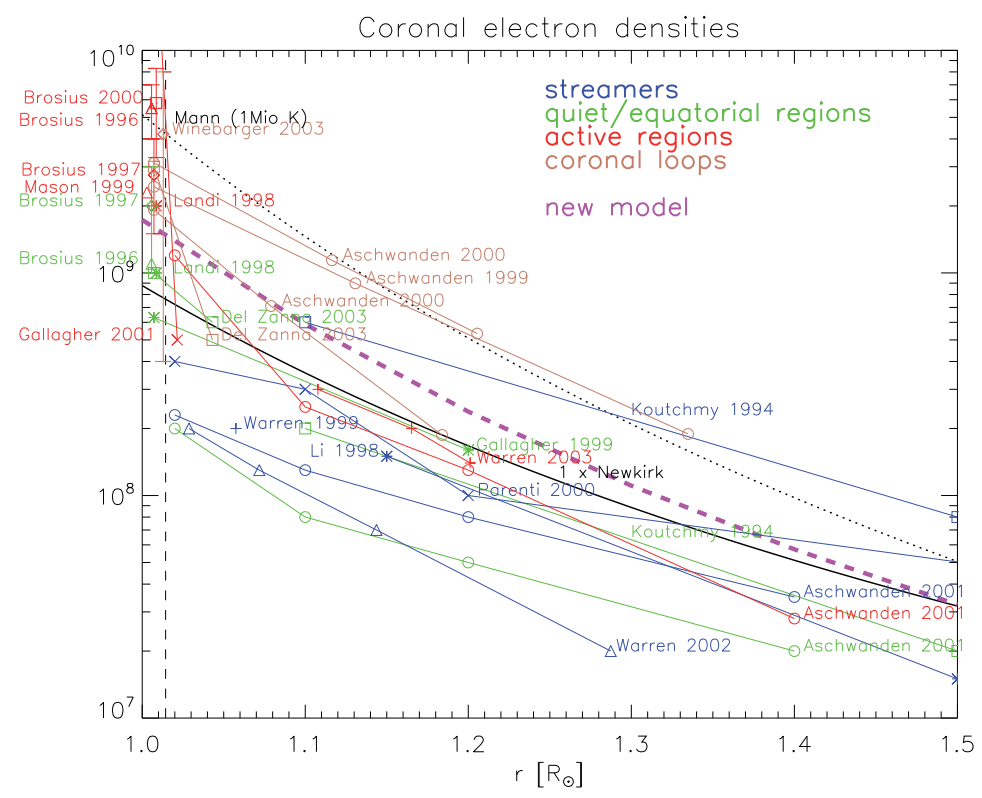

Figure 5: Electron density in inner corona above the transition region as collected by Warmuth et al. [2005] from different measurements. The new density model described here is shown by the dashed line.

\section{Results}

Fig. 6 shows the observation frequencies according to the new density model. One can see that in the region from 1 to 2 solar radii radio emission is mainly produced in the LOFAR frequency range from 30 to $240 \mathrm{MHz}$.

Applying the new electron density model to the drift rate measurement of Fig. 3 yields the radial propagation velocity $V_{r}$ shown in Fig. 7. While $V_{r}$ decreases in the inner region from 1 to 2.5 solar radii it increases beyond. It shows, that the emitting electrons have different velocities at different distances from the Sun and therefore belong to different parts of the electron distribution. Future models of the electron propagation will have to reproduce this feature.

Table 1: Comparison of parameters from literature with those from the new density model. The

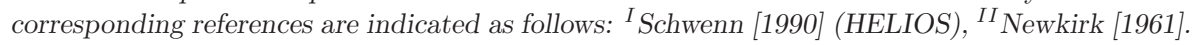

\begin{tabular}{cccccccc}
\hline \hline & $v(1 \mathrm{AU})$ & $T_{R_{\odot}}$ & $T_{1 \mathrm{AU}}$ & $N_{\mathrm{e}}\left(R_{\odot}\right)$ & $N_{\mathrm{e}}\left(1.6 R_{\odot}\right)$ & $N_{\mathrm{e}}(0.4 \mathrm{AU})$ & $N_{\mathrm{e}}(1 \mathrm{AU})$ \\
& {$[\mathrm{km} / \mathrm{s}]$} & {$\left[10^{6} \mathrm{~K}\right]$} & {$\left[10^{6} \mathrm{~K}\right]$} & {$\left[10^{8} \mathrm{~cm}^{-3}\right]$} & {$\left[10^{7} \mathrm{~cm}^{-3}\right]$} & {$\left[\mathrm{cm}^{-3}\right]$} & {$\left[\mathrm{cm}^{-3}\right]$} \\
\hline Data & $470^{I}$ & $1.0-1.5$ & $0.14^{I}$ & $8.78^{I I}$ & $2.10^{I I}$ & $48.3^{I}$ & $6.67^{I}$ \\
Model & 470 & 1.1 & 0.14 & 8.85 & 1.00 & 44.6 & 6.67 \\
\hline
\end{tabular}




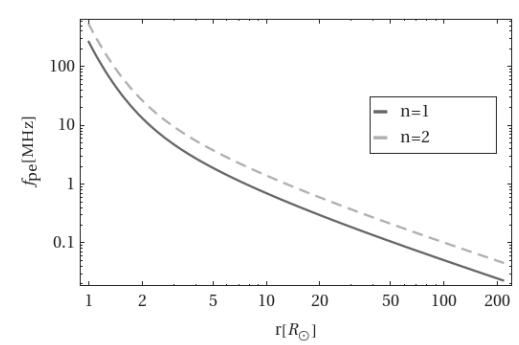

Figure 6: Observation frequency of fundamental $(n=1)$ and first harmonic $(n=2)$ plasma emission according to equation (1) and the new interplanetary density model of Fig. 4.

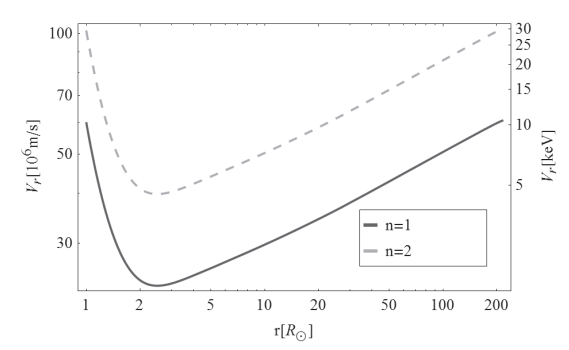

Figure 7: Radial propagation velocity $V_{r}$ (equation (3)) corresponding to the observation frequencies in Fig. 6 and the drift rate described by equation (4). The corresponding electron energies are shown to the right for comparison with in-situ measurements.

\section{Conclusion}

It was shown how information about the propagation of energetic electrons emitted by a type III burst can be obtained from dynamic radio spectra. A consistent electron density model of the plasma in the interplanetary space is necessary which was developed here. First results from its application yields a profile for the radial electron propagation velocity $V_{r}$. It shows that electrons of different velocities are responsible for the emission of radio radiation in type III burst and therefor must belong to different parts of the electron spectrum. It will be left to future work to find models of electron propagation which can resemble this feature. Next steps will also include further studies of dynamic radio spectra from solar observations with LOFAR and OSRA.

Acknowledgement. The authors want to thank Hakan Önel for his support with the computing software Wolfram Mathematica \%. His expertise was very valuable and highly appreciated.

\section{References}

Bougeret, J.-L., M. L. Kaiser, P. J. Kellogg, R. Manning, K. Goetz, S. J. Monson, N. Monge, L. Friel, C. A. Meetre, C. Perche, L. Sitruk and S. Hoang, Waves: The Radio and Plasma Wave Investigation on the Wind Spacecraft, Space Sci. Rev., 71, 231-263, 1995.

Estel, C., Ausbreitung energetischer Elektronen von der Sonnenkorona in die innere Heliosphäre, Dissertation, University Potsdam, 1999.

Gurnett, D. A., Heliospheric Radio Emissions, Space Sci. Rev., 72, 243-254, 1995. 
Hackenberg, P., E. Marsch and G. Mann, On the origin of the fast solar wind in polar coronal funnels, Astron. Astrophys., 360, 1139-1147, 2000.

Koutchmy, S., Coronal physics from eclipse observations, Adv. Space Res., 14, (4)29(4) $39,1994$.

Krall, N. A., and A. W. Tivelpiece, Principles of Plasma Physics, McGraw-Hill, New York, 1973.

Lin, R. P., and H.S. Hudson, 10 - $100 \mathrm{keV}$ electron acceleration and emission from solar flares, Solar Phys., 17, 412, 1971.

Lin, R. P., et al (65 co-authors), The Reuven Ramaty High-Energy Solar Spectroscopic Imager (RHESSI), Solar Phys., 210, 3-32, 2002.

Mann, G., H. Aurass, W. Voigt, and J. Paschke, Preliminary observations of solar type 2 bursts with the new radiospectrograph in Tremsdorf, in Coronal Streamers, Coronal Loops, and Coronal and Solar Wind Composition, ESA SP-348, 129-132, 1992.

Mann, G., F. Jansen, R. J. MacDowall, M. L. Kaiser and R. G. Sone, A heliospheric density model and type III radio bursts, Astron. Astrophys., 348, 614-620, 1999.

Melrose, D. B., Plasma emission mechanisms, in Solar Radio Physics, edited by D. J. McLean and N. R. Labrum, Cambridge University Press, Cambridge, 177-210, 1985.

Newkirk, Jr. G., The Solar Corona in Active Regions and the Thermal Origin of the Slowly Varying Component of Solar Radio Radiation, Astrophys. J., 133, 1961.

Parker, E. N., Dynamics of the interplanetary gas and magnetic fields, Astrophys. J., 128, 664-676, 1958.

Priest, E. R., Solar magneto-hydrodynamics, Dordrecht, Holland, 1982.

Schwenn, R., Large-Scale Structure of the Interplanetary Medium, in Physics of the inner heliosphere I, Springer-Verlag Berlin, 20, 99, 1990.

Suzuki, S., and G. A. Dulk, Bursts of Type III and Type V, in Solar Radio Physics edited by D. J. McLean and N.R. Labrum, Cambridge University Press, Cambridge, 289, 1985 .

Warmuth, A., and G. Mann, A model of the Alfven speed in the solar corona, Astron. Astrophys., 435, 1123-1135, 2005.

Warmuth, A., G. Mann, and H. Aurass, Modelling shock drift acceleration of electrons at the reconnection outflow termination shock in solar flares. Observational constraints and parametric study, Astron. Astrophys., 494, 677-691, 2009. 